\title{
A Case of Paratesticular Leiomyosarcoma Successfully Treated with Orchiectomy and Chemotherapy
}

\author{
Bong Suk Ko, MD ${ }^{1}$ \\ Nae Yu Kim, MD' \\ Ah Jung Ryu, MD ${ }^{1}$ \\ Dong Soon Kim, MD 1 \\ Soo Jung Gong, MD, $P h D^{1}$ \\ Dae Kyung Kim, MD, PhD² \\ Hyun Jin Son, MD, $P h D^{3}$ \\ Jung-Ae Lee, $M D, P h D^{1}$
}

Departments of ${ }^{1}$ Internal Medicine,

${ }^{2}$ Urology, and ${ }^{3}$ Pathology,

Eulji University Hospital, Eulji University

School of Medicine, Daejeon, Korea
A 50-year-old male patient presented with a right scrotal mass that had been growing rapidly for more than one year. A heterogeneous enhancing right scrotal mass $(12 \times 9 \mathrm{~cm})$ with para-aortic and peri-caval lymphadenopathies was found on abdominal computed tomography (CT). Right orchiectomy was performed and the gross finding had shown intact testis with a well-defined, huge, whitish solid mass adjacent to the testis. According to pathology, the mass was characterized as a leiomyosarcoma, grade 3 (by National Cancer Instituted [NCI] system). Therefore, the diagnosis was stage III, grade 3 paratesticular leiomyosarcoma. The patient underwent additional systemic chemotherapy using ifosfamide and adriamycin. After nine cycles of chemotherapy, positron emission tomography-CT was performed and no FDP uptake was observed. The patient has been followed up for 12 months after systemic chemotherapy, and he has maintained a complete response. We report here on a rare case of paratesticular leiomyosarcoma treated successfully with orichiectomy and additional systemic chemotherapy.

\section{Key words}

Leiomyosarcoma, Orchiectomy, Chemotherapy
Correspondence: Jung-Ae Lee, MD, PhD Devision of Hematology/Oncology, Department of Internal Medicine, Eulji University Hospital, Eulji University School of Medicine, 95 Dunsanseo-ro, Seo-gu, Daejeon 302-799, Korea Tel: 82-42-611-3161

Fax: 82-42-611-3853

E-mail: lee982023@eulji.ac.kr

Received April 22, 2011

Accepted May 24, 2011

\section{Introduction}

Leiomyosarcoma is a malignant soft tissue tumor that can arise from any tissue containing smooth muscle. However, leiomyosarcoma arising in paratesticular tissue is extremely rare and approximately only $10 \%$ of all paratesticular sarcomas are leiomyosarcomas [1]. The primary treatment for this tumor is radical orchiectomy with high cord ligation; however, the optimal local and systemic treatment remains controversial. Adjuvant radiotherapy is generally recommended for lowering the rate of local recurrence and the role of chemotherapy has not been fully established [2]. We report here on a case of stage III paratesticular leiomyosarcoma, which was treated successfully with orchiectomy and systemic chemotherapy. 

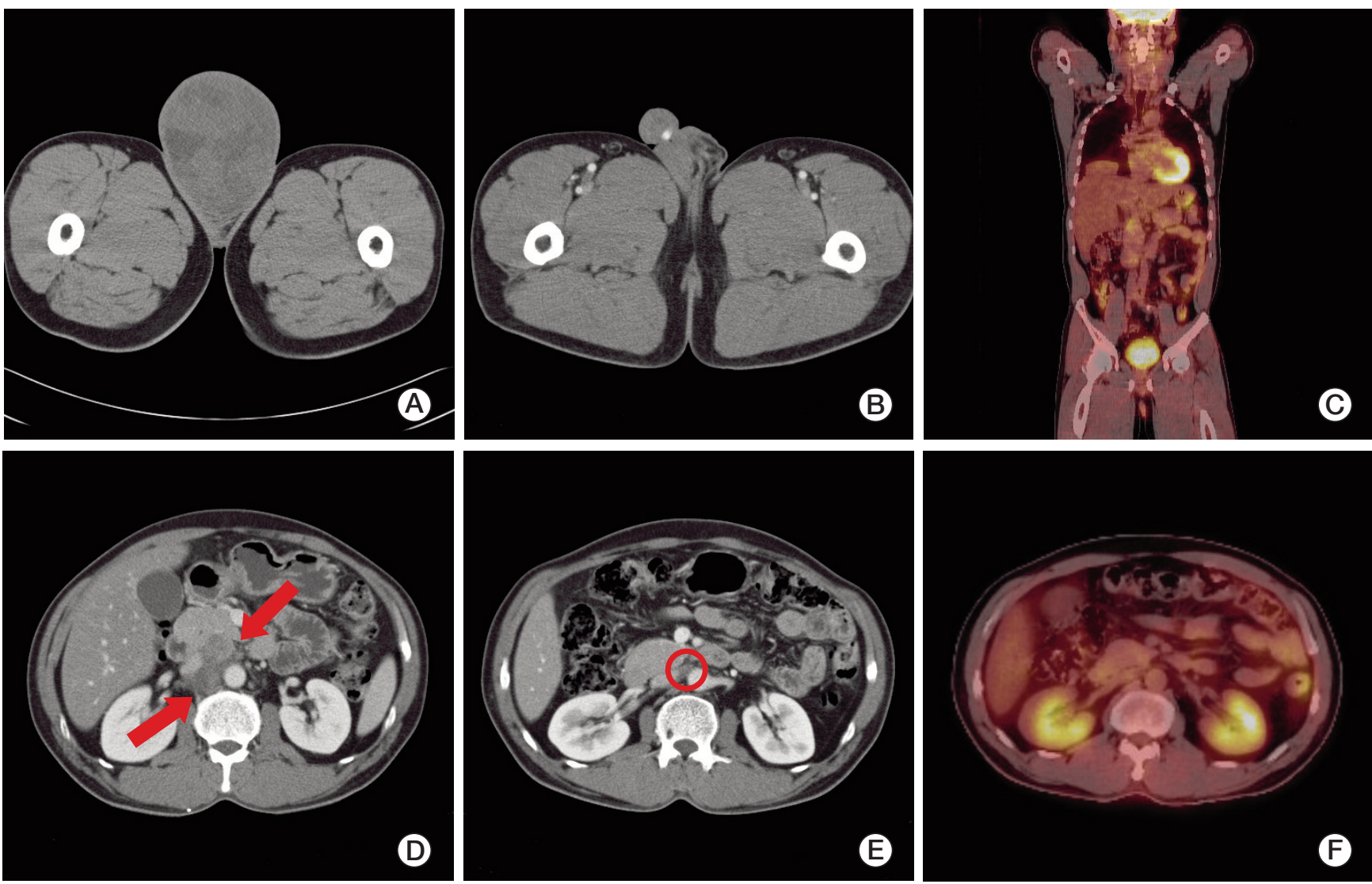

Fig. 1. (A, D) Abdominal enhanced computed tomography (CT) at the time of the first diagnosis. (A) A huge heterogeneous enhancing mass $(12 \times 9 \mathrm{~cm}$ ) in the right scrotum. (D) Multiple enlarged (about $2 \mathrm{~cm}$ ) lymphadenopathies at the para-aortic and peri-caval areas (arrows). (B, E) Abdominal enhanced CT after four cycles of chemotherapy. (B) The orchiectomy site showing no evidence of tumor recurrence. (E) The lymphadenopathy has decreased in size to less than $1 \mathrm{~cm}$ (circle). (C, F) Whole body positron emission tomography-CT after nine cycles of chemotherapy. (C) Coronal view without fructose-1,6-bisphosphate (FDP) uptake. (F) Axial view showing the significant decrease in size of lymph nodes without FDP uptake.

\section{Case Report}

A 50-year-old male patient with a 10-year history of a huge right scrotal mass, which had shown rapid growth for more than one year, was referred to our department. Results of the physical examination revealed a right paratesticular mass measuring approximately $12 \mathrm{~cm}$ in diameter, the mass was firm and non-tender. The serum lactate dehydrogenase level was 313 $\mathrm{IU} / \mathrm{L}$, the $\alpha$-fetoprotein level was $5.9 \mathrm{ng} / \mathrm{mL}$, and the $\beta$-human chorionic gonadotropin level was $1.7 \mathrm{mIU} / \mathrm{mL}$, which were all within the normal ranges. Findings on abdominal computed tomography (CT) showed a huge heterogeneous enhancing right scrotal mass $(12 \times 9 \mathrm{~cm})$ with paraaortic and peri-caval lymphadenopathies (Fig. 1A and D). Right orchiectomy was performed and the macroscopic pathologic features showed a grossly intact testis with a well-defined, huge, whitish solid mass adjacent to the testis (Fig. 2). Immunohistochemical staining showed a positive result for smooth muscle actin (SMA), CD34, and desmin; however, immunostaining for myoglobin, S-100 protein, and c-kit showed a negative result. According to the pathologic results, the mass was

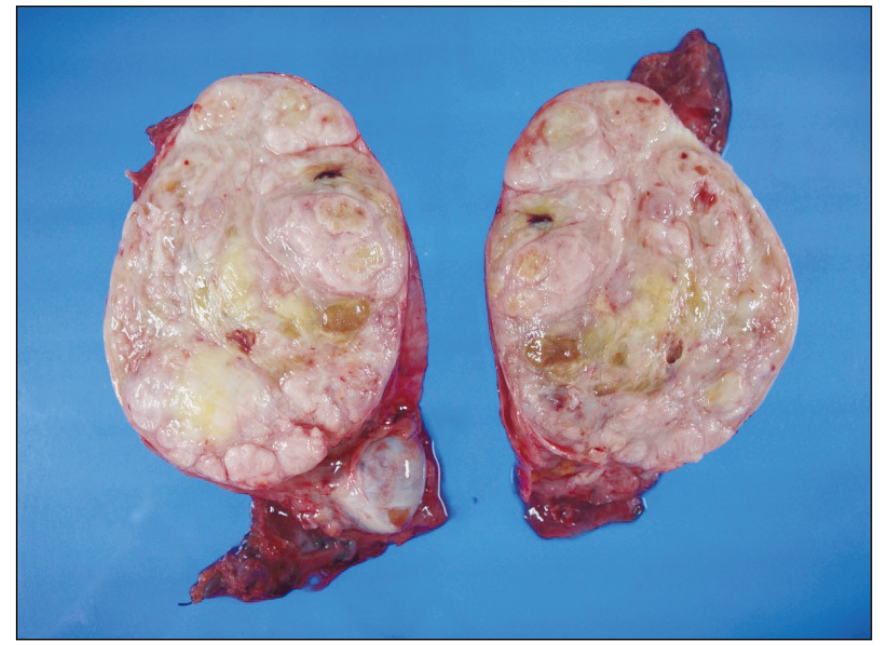

Fig. 2. Gross findings. A well-defined huge solid mass $(13 \times 10 \mathrm{~cm})$ is seen adjacent to the testis. The testis is of normal size and is grossly intact. The tumor is completely separated from the testis and has a whitish tan, solid and trabeculated cut surface. 

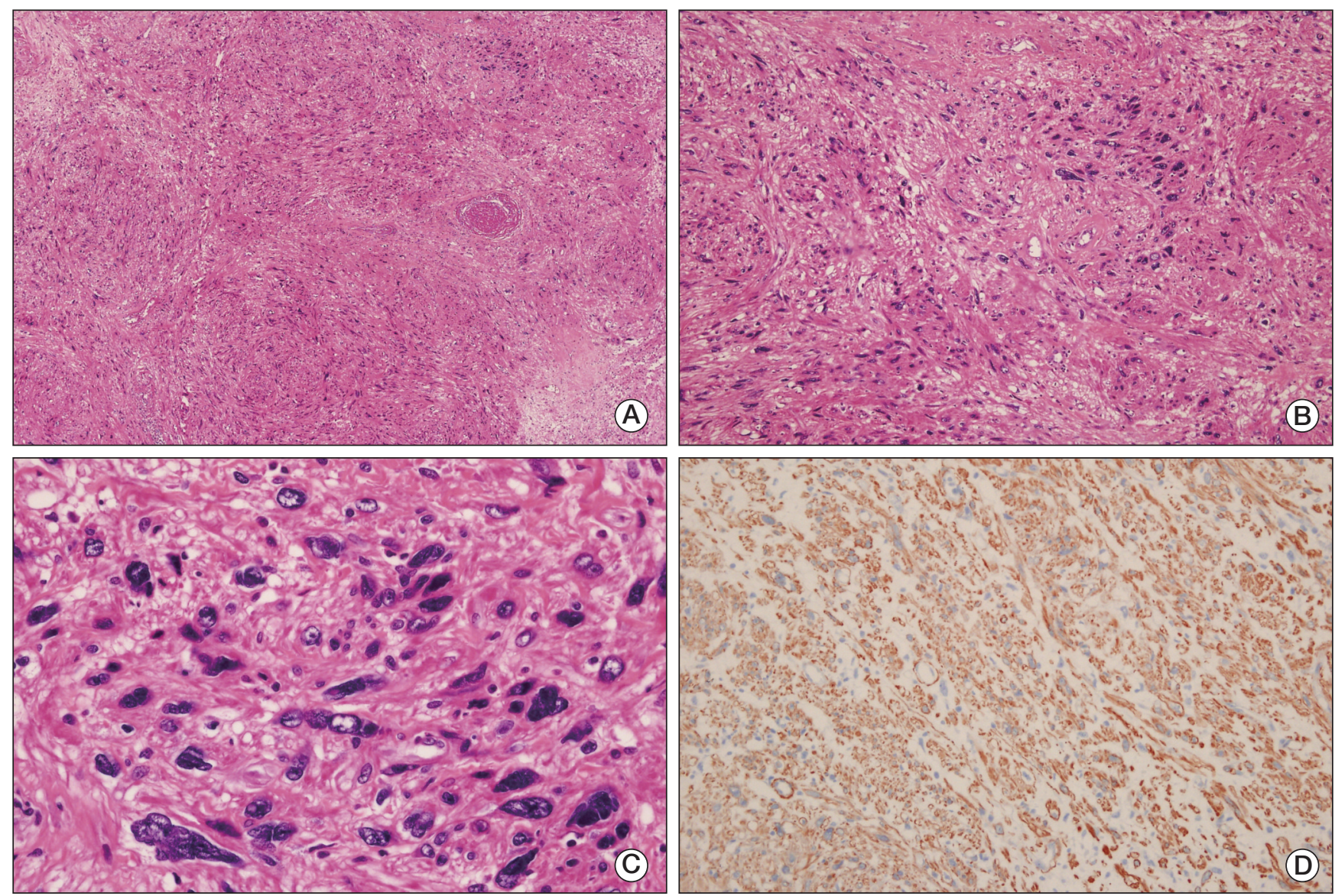

Fig. 3. Microscopic findings. (A) The lower power view shows tumor cells arranged in a whorled or intersecting pattern and a partially necrotic portion is identified (H\&E staining, $\times 12.5$ ). (B) The tumor shows high atypism and is composed of spindle cells, indicating their probable mesenchymal origin (H\&E staining, $\times 100$ ). (C) The high power view displays the nuclear pleomorphism and many mitoses of the tumor cells (H\&E staining, $\times 400)$. (D) Immunohistochemical staining shows diffuse and strong positivity for smooth muscle actin $(\times 200)$.

characterized as a leiomyosarcoma, grade 3 (according to the French National Federation of Cancer Centers [FNCLCC] and National Cancer Institute [NCI] system): the total score was 8 , the mitotic score was 3 (more than 20/10 high power field), the necrosis score was 2 (more than $50 \%$ ), and the differentiation score was 3 (Fig. 3). According to the American Joint Committee on Cancer Sarcoma Staging system, this patient was stage III (T2aN1M0), therefore, we diagnosed the patient as having a stage III, grade 3 paratesticular leiomyosarcoma. The patient underwent additional systemic chemotherapy using ifosfamide $1 \mathrm{~g} / \mathrm{m}^{2}$ on days 1 to 3 plus adriamycin $30 \mathrm{mg} / \mathrm{m}^{2}$ on day 1 . Chemotherapy cycles were repeated every 21 days. After four cycles of chemotherapy, we performed abdominal CT for evaluation of the therapeutic response. The lymphadenopathies that were initially observed in the abdominal cavity had decreased in size by over $50 \%$ with no tumor recurrence, therefore, a partial response could be identified (Fig. 1B and E). After nine cycles of chemotherapy, whole body positron emission tomography-CT was performed and no uptake of fructose-1,6-bisphosphate (FDP) was observed (Fig. 1C and F). The patient has been followed up for 12 months after systemic chemotherapy, and he has consistently maintained a complete response (CR).

\section{Discus sion}

Leiomyosarcoma is a soft tissue tumor arising from smooth muscle cells of a mesenchymal origin. Paratesticular leiomyosarcoma originates from the spermatic cord, the scrotum, or the epididymis. The most common type is the spermatic cord type, which arises from undifferentiated mesenchymal cells of the cremasteric muscle and the vas deferens. The epididymal and scrotal types are less frequent and they originate from the smooth muscle surrounding the basement membrane of the epididymal canal and dartous layer, respectively [3]. Leiomyosarcomas have three typical histological features; these include perpendicularly arranged fascicles of spindle cells with eosinophilic cytoplasm, hyperchromatic blunt-ended nuclei, and scattered paranuclear vacuoles [4]. On immunohistochemical staining, expression of SMA, muscle specific actin, and desmin is observed in most leiomyosarcomas, while expression of CD34, myogenin, Ki-67, S-100 protein, and cytokeratin has also been reported in some cases [5]. This case showed a positive result for SMA, CD34, and desmin. Paratesticular leiomyosarcoma presents as a discrete nodular 
mass, which is completely separate from the testicle [2]. Although the etiology of leiomyosarcomas remains unclear, some authors have suggested local irradiation during childhood as a potential cause [6]. Clinically, most paratesticular leiomyosarcomas present as painless, slow-growing scrotal tumors in men of middle or older age. Occurrence of this disease is rare; therefore, the mode of spread is important. The most common means of spread is lymphatic, followed by hematogenous, and, last, by local extension. The route of lymphatic dissemination may involve the external iliac, hypogastric, common iliac, and para-aortic nodes. The lung is the primary site of blood-borne metastases. Local spread to the scrotum, inguinal canal, or pelvis along the pathway of the vas deferens is possible [3].

Paratesticular leiomyosarcoma is very rare and accumulation of a sufficient number of cases in order to document the natural history of such tumors along with treatment is difficult. Therefore, most documented treatments for paratesticular leiomyosarcoma have been categorized together with those for other paratesticular sarcomas. In general, the standard treatment for all paratesticular sarcomas consists of radical orchiectomy with high cord ligation. The role of additional systemic chemotherapy is not clear. However, paratesticular leiomyosarcoma has recently been classified according to the NCI system in order to determine the correlation between the tumor grade and clinical outcomes. According to one report, paratesticular leiomyosarcomas that were pathologic grade 1 and 2 (according to the NCI system) and that were treated with radical orchidectomy were free of recurrence and metastasis during the follow up period [5]. Although data from that report were insufficient, the importance of tumor grading for leiomyosarcoma in order to further assess the efficacy of various therapeutic options was demonstrated. Adjuvant therapy has not been well established, however, in earlier reports, adjuvant radiation therapy was usually recommended in order to reduce locoregional recurrence [7]. Although the role of adjuvant chemotherapy has not been fully established, some studies have reported on this. Findings of a meta-analysis of 14 randomized trials of sarcomas at various centers showed that doxorubicin-based adjuvant chemotherapy resulted in significant improvement of the time to local and distant failure, and, although it was not statically significant, a trend toward improved overall survival was observed [8]. In another study of adult sarcomas at different sites, doxorubicin based chemotherapy resulted in an increase in overall and metastasis free survival in patients with grade 3 sarcomas [9]. In our case, the tumor was NCI grade 3, and, after undergoing right orchiectomy, the patient received additional systemic chemotherapy using ifosfamide $1 \mathrm{~g} / \mathrm{m}^{2}$ on days 1 to 3 plus adriamycin $30 \mathrm{mg} / \mathrm{m}^{2}$ on day 1 every three weeks. While undergoing systemic chemotherapy, the patient did not complain of any side effects and there was no bone marrow suppression, such as leukocytopenia. After nine cycles of systemic chemotherapy, the patient achieved a CR, and neither local recurrence nor distant metastases has occurred during the follow up period (12 months) after completion of systemic chemotherapy.

Due to the low number of reported cases of additional systemic chemotherapy for treatment of patients with stage III paratesticular leiomyosarcoma, the role of systemic chemotherapy has not been well established. And few domestic cases of paratesticular leiomyosarcoma have been reported [10-14], however, those cases were treatable with curative resection and the effect of chemotherapy against stage III paratesticular leiomyosarcoma was not documented, as in our case. In one retrospective analysis of the outcome of first-line chemotherapy in patients with male non-uterine advanced or metastatic leiomyosarcoma, median progression free and overall survival were 2.7 and 7.9 months, respectively [15]. As a result of successful treatment, this current case could be unique, and, although our case had a short-term follow up period of 12 months after systemic chemotherapy, 19 months disease free survival seems remarkable, compared with other cases. Longer term follow up is needed; therefore, the final results are still inconclusive.

In conclusion, this is a rare case of paratesticular leiomyosarcoma treated with orchiectomy and systemic chemotherapy. The role of chemotherapy in treatment of paratesticular leiomyosarcoma has not been well established, and this is still is a matter of discussion. However, this case has shown successful results with ifosfamide and adriamycin for treatment of stage III disease. Therefore, we report here on a case of paratesticular leiomyosarcoma treated successfully with orchiectomy and chemotherapy.

\section{Conflicts of Interest}

Conflicts of interest relevant to this article was not reported.

\section{References}

1. Lopes RI, Leite KR, Lopes RN. Paratesticular leiomyosarcoma treated by enucleation. Int Braz J Urol. 2006;32:66-7.

2. Khoubehi B, Mishra V, Ali M, Motiwala H, Karim O. Adult paratesticular tumours. BJU Int. 2002:90:707-15

3. Dangle P, Basavaraj DR, Bhattarai S, Paul AB, Biyani CS. Leiomyosarcoma of the spermatic cord: case report and literature review. Can Urol Assoc J. 2007;1:55-8.

4. Hashimoto H, Daimaru Y, Tsuneyoshi M, Enjoji M. Leiomyosarcoma of the external soft tissues: a clinicopathologic, immunohistochemical, and electron microscopic study. Cancer. 1986:57:2077-88.

5. Fisher C, Goldblum JR, Epstein Jl, Montgomery E. Leiomyosarcoma of the paratesticular region: a clinicopathologic study. Am J Surg Pathol. 2001;25:1143-9.
6. Dalton DP, Rushovich AM, Victor TA, Larson R. Leiomyosarcoma of the scrotum in a man who had received scrotal irradiation as a child. J Urol. 1988;139:136-8.

7. Fagundes MA, Zietman AL, Althausen AF, Coen JJ, Shipley WU. The management of spermatic cord sarcoma. Cancer. 1996;77:1873-6.

8. Adjuvant chemotherapy for localised resectable soft-tissue sarcoma of adults: meta-analysis of individual data. Sarcoma Meta-analysis Collaboration. Lancet. 1997;350:1647-54.

9. Coindre JM, Terrier P, Bui NB, Bonichon F, Collin F, Le Doussal V, et al. Prognostic factors in adult patients with locally controlled soft tissue sarcoma: a study of 546 patients from the French Federation of Cancer Centers Sarcoma Group. J Clin Oncol. 1996;14:869-77.

10. Chung HC, Lee HS, Kim TI, Eom MS, Song JM. Local recurrence of spermatic cord leiomyosarcoma. Korean J Urol. 2009;50:92-5. 
11. Kim BK, Chang HS, Park CH, Kim Cl. Paratesticular leiomyosarcoma. Korean J Urol. 2005;46:426-9.

12. Yoon CY, Kwon SD, Cho JH, Kim CW. A case of leiomyosarcoma of the scrotum. Korean J Urol. 1996:37:1421-3.

13. Ahn JS, Kim HJ, Kim YG, Park YK. A case of leiomyosarcoma of spermatic cord. Korean J Urol. 1992:33:904-6.
14. Jo EJ, Kang SJ, Han CS, Shin SJ, Choi SH. A case of leiomyosarcoma of the spermatic cord. Korean J Urol. 1989:30:274-6.

15. Oosten AW, Seynaeve C, Schmitz PI, den Bakker MA, Verweij J, Sleijfer S. Outcomes of firstline chemotherapy in patients with advanced or metastatic leiomyosarcoma of uterine and non-uterine origin. Sarcoma. 2009;2009:348910. 